\title{
Research Paper \\ Comparing Enamel Microhardness in Decidous Teeth With Primary Carious Lesions After Applying Three Fluoride Containing Materials in Vitro
}

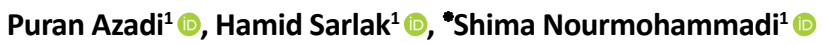

1. Department of Pediatric Dentistry, Faculty of Dentistry, Arak University of Medical Science, Arak, Iran.

\begin{tabular}{|l|l|l}
\hline $\begin{array}{c}\text { Use your device to scan } \\
\text { and read the article online }\end{array}$ & $\begin{array}{l}\text { Cftation: Azadi P, Sarlak H, Nourmohammadi Sh. [Comparing Enamel Microhardness in Decidous Teeth With Primary Cari- } \\
\text { ous Lesions After Applying Three Fluoride Containing Materials in Vitro (Persian)]. Journal of Arak University of Medical Sci- } \\
\text { ences (JAMS). 2021; 24(2):256-267. https://doi.org/10.32598/JAMS.24.2.6175.1 }\end{array}$ \\
dol'https://doi.org/10.32598/JAMS.24.2.6175.1
\end{tabular}

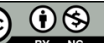

Article Info:

Received: 03 May 2020

Accepted: 29 Nov 2020

Available Online: 01 Jun 2021

\section{A B STRACT}

Background and Aim For decades, using fluoride has been introduced to prevent the development and progression of primary carious lesions. Increased surface microhardness of primary caries is among the essential factors in preventing lesion progression and cavity formation. The present study aimed to compare the microhardness changes of primary caries treated with 3 products, containing fluoride (varnish, toothpaste, \& mouthwash) in dental teeth.

Methods \& Materials In this study, 45 extracted human deciduous molars were used. Using a diamondwinning disc, enamel blocks with a dimension of $1 \times 4 \times 4 \mathrm{~mm}$ were prepared from the buccal surface of the teeth, i.e., healthy and without structural defects; they were mounted in acrylic self-adhesive. Initial microhardness test (Vicker's test) with a force of $300 \mathrm{gr}$ and Dwell time for 15 seconds was applied on samples. Next, artificial caries with the standard solution were created in all samples and the microhardness of samples was recorded at this stage. The study samples were randomly divided into 3 groups of 15, treated with fluoride varnish, mouthwash, and toothpaste. Then, the microhardness of samples was re-measured. The mean surface microhardness was compared between the study groups by Independent Samples t-test and Bonferroni test. All analyses were performed using SPSS at the significance level of $\mathrm{P}<0.05$.

Ethical Considerations This study was approved by the Ethics Committee of the Arak University of Medical Science (Code: IR.ARAKMU.REC.1397.264).

Results Increase in surface microhardness of primary caries in fluoride varnish group was statistically significant; in GC MI paste plus toothpaste and Oral B mouthwash was non-significant. The research samples treated by fluoride varnish had a higher surface microhardness, compared to toothpaste and mouthwash. Conclusion According to the present research results, fluoride varnish was superior to fluoride mouthwash and toothpaste in improving the microhardness of primary dental caries.
Enamel microhard-

ness, Fluoride,

Remineralization tion in receiving treatment is poor, is always a challenge for dentists. Due to the different structure of deciduous teeth, compared to permanent teeth and the rapid progression of caries as a result of pulp involvement, always early and noninvasive treatment methods are of high priority to prevent caries or stop the process of caries in the early stages (Early/

\section{Extended Abstract}

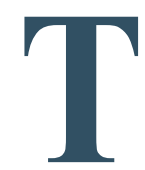

\section{Introduction}

reating deciduous teeth in children, especially at a young age when their coopera-
* Corresponding Author:

\section{Shima Nourmohammadi, PhD.}

Address: Department of Pediatric Dentistry, Faculty of Dentistry, Arak University of Medical Science, Arak, Iran.

Tel: +98 (912) 4600879

E-mail: shima.nourmohammadi@arakmu.ac.ir 
White spot lesions) in children [12]. Numerous laboratory studies compared the effects of different substances on the enhancement of enamel microhardness; however, most studies were performed on permanent human or animal teeth. Deciduous enamel is different from permanent teeth concerning microscopic structure. Thus, the present study aimed to compare the effects of three fluoride-containing substances on microhardness changes in primary decay enamel of deciduous teeth artificially and under laboratory conditions.

\section{Materials and Methods}

In this study, 45 extracted human deciduous molars were used. The maximum storage time of the samples was less than 3 months. The collected samples were immersed in $0.1 \%$ thymol solution for 24 hours for disinfection. Using a brush with a slow handpiece, they were cleaned of any debris and washed with distilled water, and remained at room temperature until studied in normal saline solution. Using a diamond cutting disc, enamel blocks with dimensions of $1 \times 4 \times 4 \mathrm{~mm}$ were prepared from the buccal surface and mounted on Acropars self-healing acrylic. The obtained samples were divided into 3 groups of 15 (first group: treated with fluoride varnish, second group: treated with fluoride mouthwash, \& the third group was treated with fluoride toothpaste) and the microhardness of each group was taken in 3 steps, as follows:

Step 1: Measure the initial microhardness of the samples
Step 2: Measure the microhardness of the samples of all three groups after causing artificial caries

Step 3: Measure the microhardness of the samples in each group after exposure to fluoride-containing materials

\section{Results}

Based on the present study findings, the mean microhardness (in all 3 groups), significantly decreased after artificial caries, compared to healthy enamel $(\mathrm{P}<0.05)$.

The mean microhardness of healthy enamel in the first group was measured to be 347 VH89 and VH37/257 after artificial rot. It was found to indicate an increase in microhardness in this group. There was a significant difference between the mean microhardness of the first and second stages of measurement as well as the second and third stages of measurement; however, there was no significant difference between the mean value of the first and third stages of measurement, highlighting the positive effect of fluoride varnish in increasing enamel remineralization. Artificial decay was to such an extent that it was not significantly different from healthy enamel $(\mathrm{P}=0.126)$.

In the second group, the mean microhardness of healthy enamel equaled $313.89 \mathrm{VH}$, which decreased to 254.69 VH after artificial caries; after receiving mouthwash twice a week for 3 weeks, the mean microhardness of samples increased to $268 / 67 \mathrm{VH}$. There was a significant difference between the microhardness measured in the first and

Table 1. Mean microhardness value in the fluoride varnish group in the stages of healthy enamel, artificial decay, and fluoride varnish (VH)

\begin{tabular}{|c|c|}
\hline Group & Mean \pm SD \\
\hline Healthy enamel & $347.85 \pm 48.98$ \\
\hline Artificial cavity & $257.37 \pm 42.87$ \\
\hline VH & $329.30 \pm 42.84$ \\
\hline
\end{tabular}

Table 2. Mean microhardness value in the mouthwash group in the stages of healthy enamel, artificial decay, and fluoride varnish (VH)

\begin{tabular}{cc}
\hline Group & Mean \pm SD \\
\hline Healthy enamel & $313.89 \pm 43.58$ \\
Artificial cavity & $254.69 \pm 56.64$ \\
Fluoride mouthwash & $267.67 \pm 40.73$ \\
\hline
\end{tabular}


Table 3. Mean microhardness value in the toothpaste group in the stages of healthy enamel, artificial decay, and fluoride varnish (VH)

\begin{tabular}{cc}
\hline Group & Mean \pm SD \\
\hline Healthy enamel & $331.45 \pm 68.44$ \\
\hline Artificial cavity & $255.90 \pm 55.78$ \\
Fluoride mouthwash & $263.27 \pm 42.35$ \\
\hline
\end{tabular}

second, as well as first and third stages; however, despite the increase in microhardness after the effect of Oral B $0.12 \%$ mouthwash, no significant difference was observed between the microhardness measured in the second and third stages. In the third study group, measurements were performed as per the previous two groups and the average microhardness of healthy enamel was computed as 331.45 $\mathrm{VH}$, which decreased to $255.90 \mathrm{VH}$ after artificial caries; after using toothpaste, the average microhardness of the study samples reached $263 / 27 \mathrm{VH}$ daily for 20 days. In this group, as in the second group, there was a significant difference between the microhardness measured in the first and second, as well as first and third stages; however, despite the increase in microhardness after the effect of $0.2 \%$ GC MI Paste plus $0.2 \%$ toothpaste between the measured microhardness, there were no significant differences between the second and third stages. The effect of V-Varnish fluoride $5 \%$ on increasing the microhardness of enamel with artificial decay was significant i.e., higher than Oral B mouthwash $0.12 \%$ and GC MI Paste plus toothpaste $0.2 \%$. Microhardness changes between toothpaste and mouthwash groups were not statistically significant.

\section{Discussion}

In this laboratory study, microhardness changes in the primary enamel of deciduous teeth (artificially created) were investigated after the application of $\mathrm{V}$-varnish fluoride varnish, Oral B Complete mouthwash, and GC MI Paste plus toothpaste. The purpose of the demineralization and remineralization cycle in vitro is to evaluate the effectiveness of fluoride-containing compounds to protect the demineralized enamel against acid [14]. The process used in this study was a standard formula to simulate the condition of the mouth that acidic substances and the activity of cariogenic bacteria that cause the demineralization of tooth enamel and primary caries (White Spot Lesion).

Due to the desirable properties of fluoride varnish, including its simple use for young children (even before the age of one year), the small volume of the material used, reducing the possibility of swallowing and no need to maintain isolation after application on the teeth and due to the higher concentration of fluoride in the varnish than toothpaste and mouthwash, its use is recommended three times a year for children at high risk of caries or a three-course diet with 10-day intervals within a month. Also, due to the increased microhardness of enamel with primary decay after using mouthwash, it is recommended to use mouthwashes containing fluoride after the age of 6 years. According to the present study, the least improvement in microhardness was observed in the group of toothpaste. However, due to the increase in microhardness of enamel with primary decay and the use of a toothbrush with toothpaste is the most common method of plaque control and oral hygiene at home. It is also recommended to use a regular fluoride toothpaste in children after 2-3 years of age.

\section{Ethical Considerations}

\section{Compliance with ethical guidelines}

This study was approved by the Ethics Committee of the Arak University of Medical Science (Code: IR.ARAKMU. REC.1397.264).

\section{Funding}

This research did not receive any grant from funding agencies in the public, commercial, or non-profit sectors.

\section{Authors' contributions}

All authors equally contributed to preparing this article.

\section{Conflicts of interest}

The authors declared no conflicts of interest. 


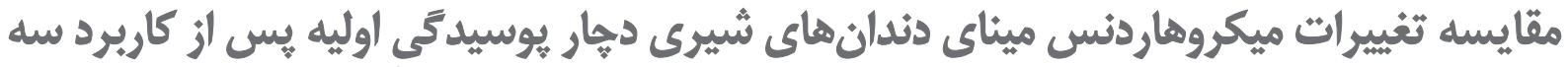

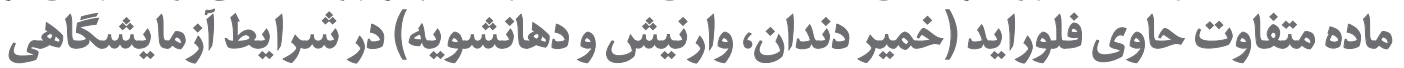

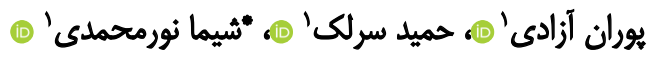

1. كروه دندانيزشكى كودكان، دانشكده دندانيزشكى، دانشكاه علوم يزشكى اراك، اراك، ايران.

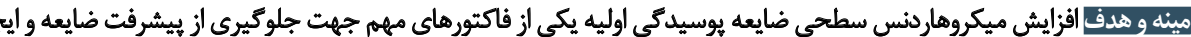

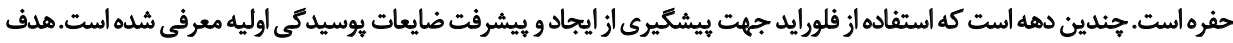

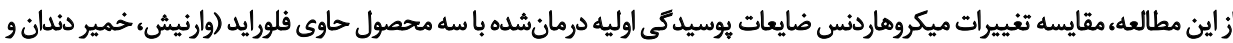

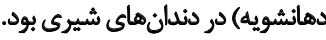

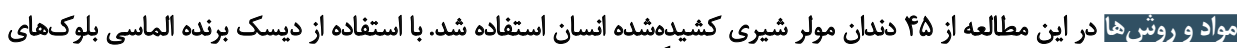

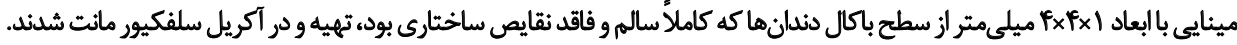

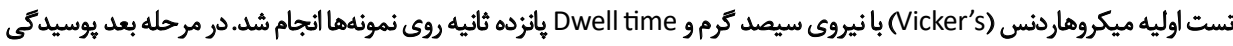

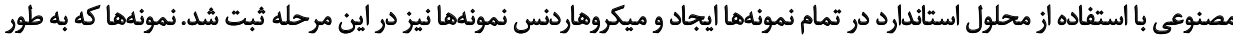

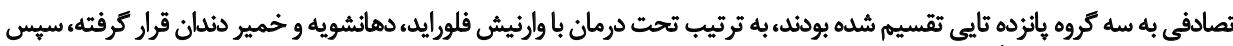

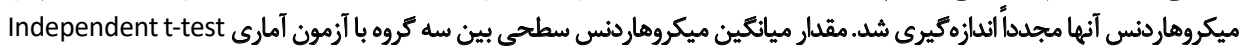

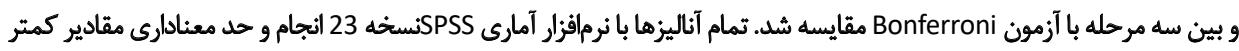

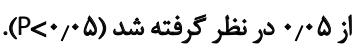

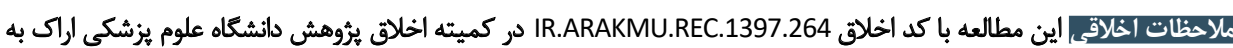

ثبت رسيدهاست.

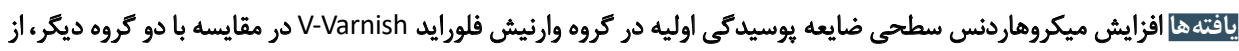

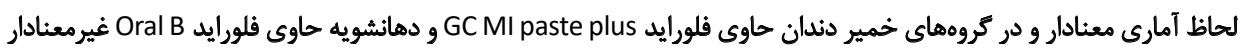

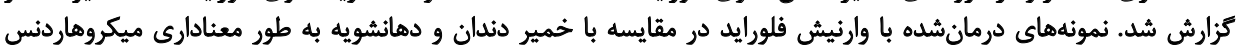
سطحى بالاترى داشتيند.

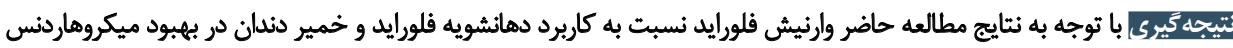

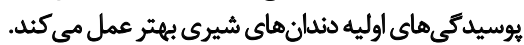

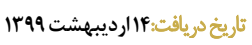

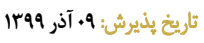

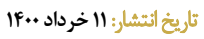

كليدوأوهها:

ميكروهاردنس مينا، فلورايد، رمينراليزاسيون

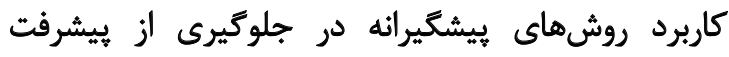

dôم

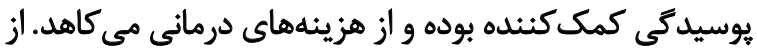

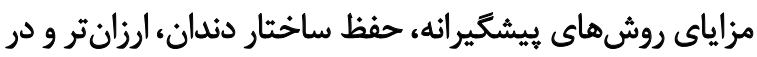

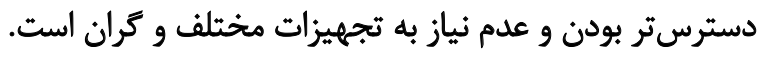
در مطالعات آزمايشعاهى و بالينى بسيارى از محصولات

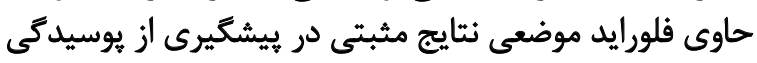

يوسيدكى دندان يكى از شايعترين بيمارى هاى مزمن جارئ جهان

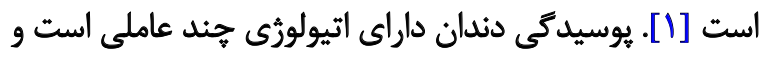

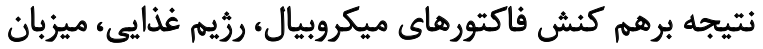


(1) سيستميك: با قرار گرفتن در ساختار مينا حين تشكيل

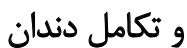

r) موضعى: باكمك به رمينراليزاسيون و مهار دمينراليزاسيون

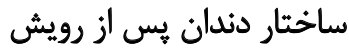

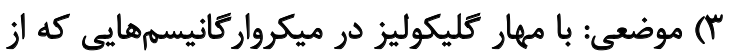

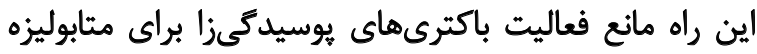
كردن كربوهيدراتها و توليد اسيد مي فئرد.

وارئيشهاى فلورايد موادى هستند كه داراى ماده زمينهاى

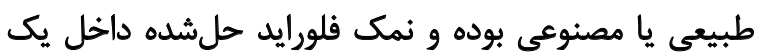

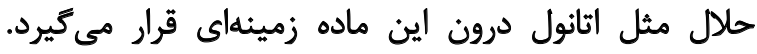

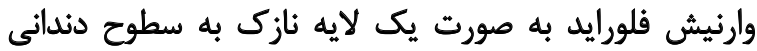

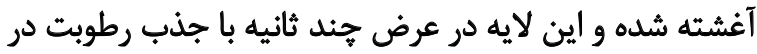

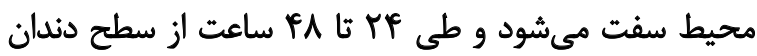

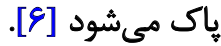

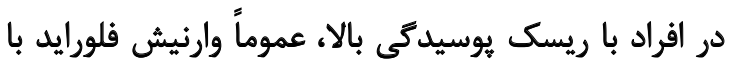

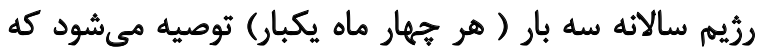

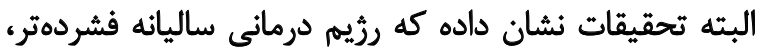

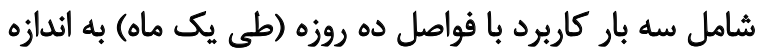

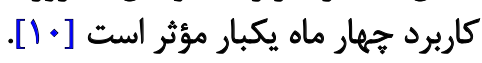

اصطلاح خمير دندانَ از دو كلمه Dens به معند معناى دندان و

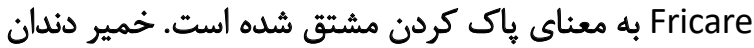

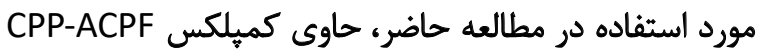

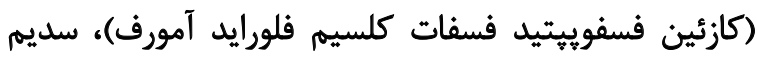

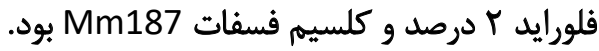
اعمال خمير دندانها عبارتاند از: سفيدكنئدكى، پإليش

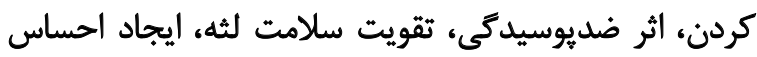
تميزى و كنترل بوى بد دهان. دهانشويهها" محصولات كمكى جهت ارتقاى سلامت دهان

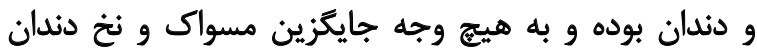

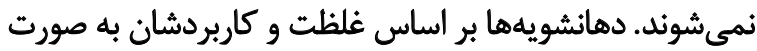

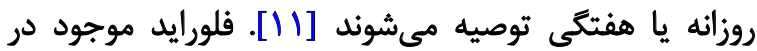
دهانشويه، سديم فلورايد ب درصي توصد است. دهانشويهها انواع مختلف دارد: () دهانشويه آنتىباكتريال (جهت بهببود وضعيت لثه و بافت

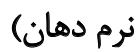

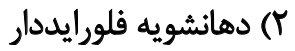

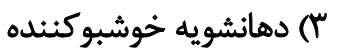

دندان نشان دادهاند [ب[]. روند ايجاد يوسيدگى فرايندى يويا

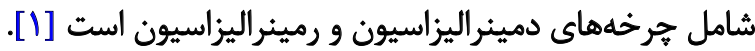
در روند يوسيدكى ثاج، بافت سخت دندان (مينا و سيس توسيس

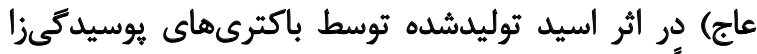

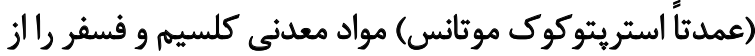

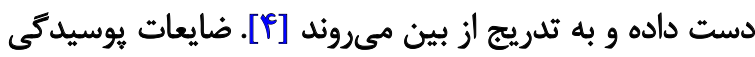

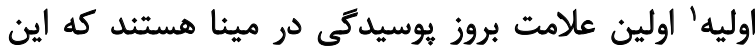

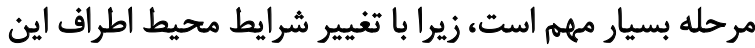

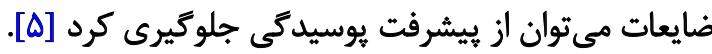

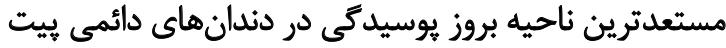

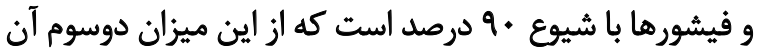

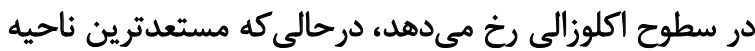

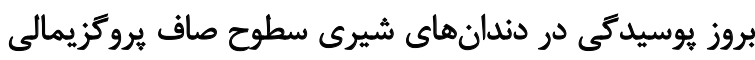

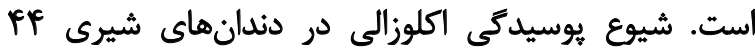
درصد است [ب].

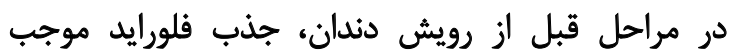

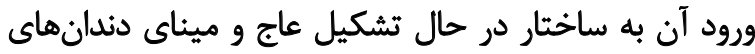

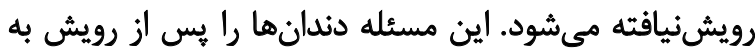

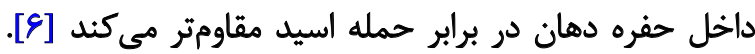

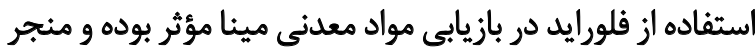
به افزايش ميكروهاردنس مينا مىشود [V] اثرات فلورايد هم به صورت موضعى و هم سيستميك

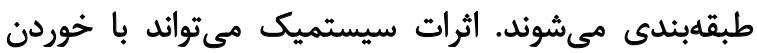

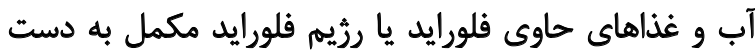

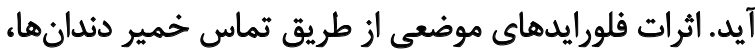

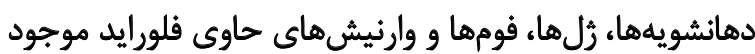

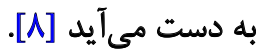

مينا توسط سلولهايى كه منشأ آنها لايه جنينى اكتودرم

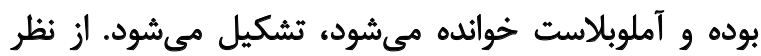

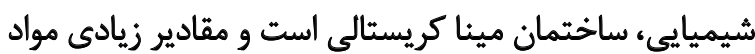

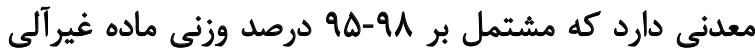

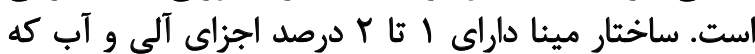
حدود f اسر دصد وزنى است.

مينا داراى ميليونها منشور مينايى است كه بزركت مثرين جزء

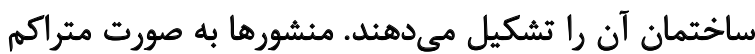

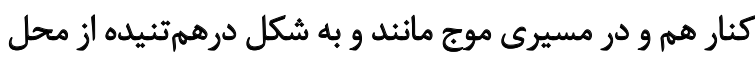

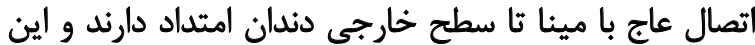

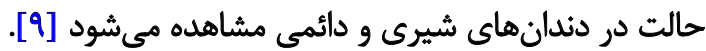
فلورايد از سه طريق موجب بيشغيرى از يوسيدكى دندانى و وني

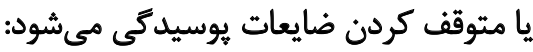

1. White Spot 
در هر ناحيه يك بار تست ميكروهاردنس با روش Kicker's ter (Koopa hardness tester microhardness انجام كرفته كه type,Model:MH2.1.2-Koopa-Iran) فرورونده الماسى كار كذاشته شده در دستكاه در ميناي دندان

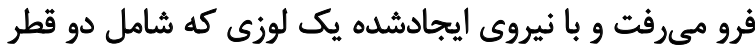

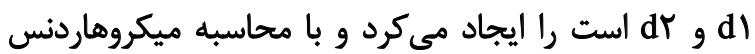

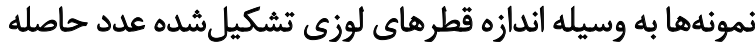

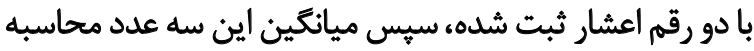

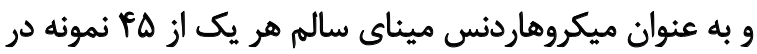

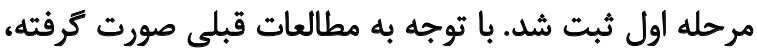

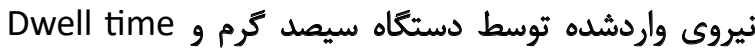

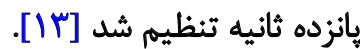

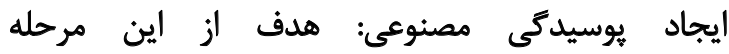

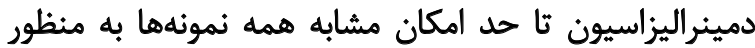

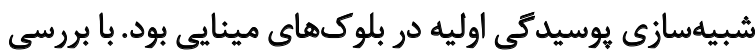

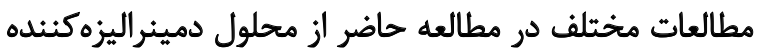

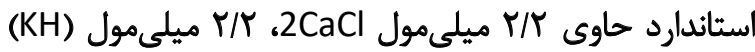
TFPO

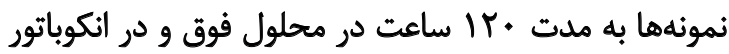

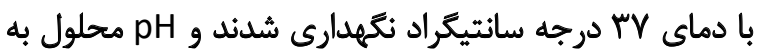

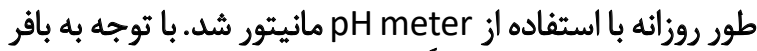

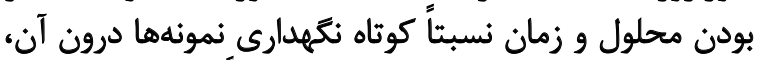

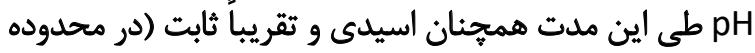

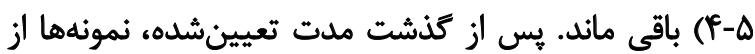

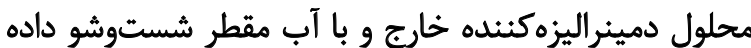

شدند.

مرحله دوم سنجش ميكروهاردنس: در اين مرحله در يك

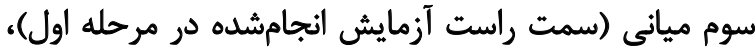

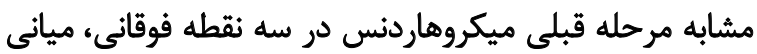

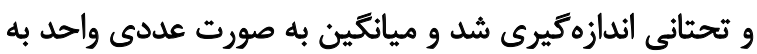

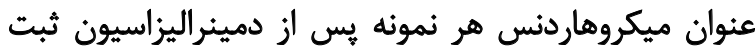

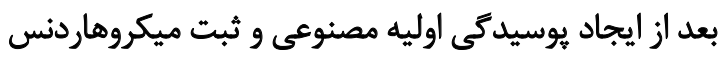

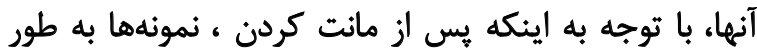

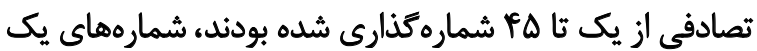

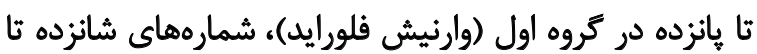

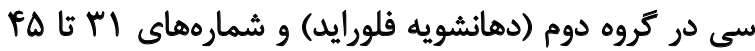
در تروه سوم (خمير دندان فلورايد دار) قرار ترفيتند.

كروههاى مورد مطالعه به صورت زير بودند:

كروه اول: سطح نمونهها بس از شستوشو با بـ آب مقطر با فشار

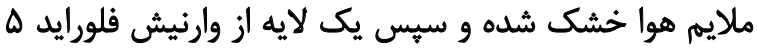

درمان دندانهاى شيرى در كودكان، بهخصوص در سنين

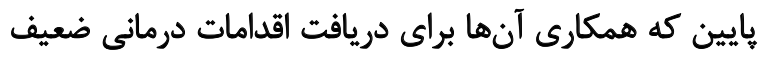

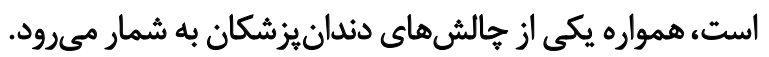
با توجه به ساختار متفاوت دندانهاى شيرى نسبت به ديه

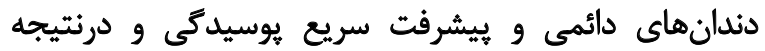

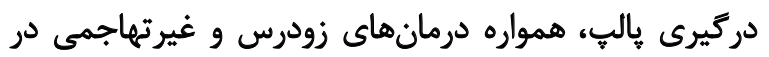

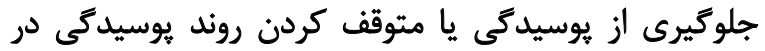

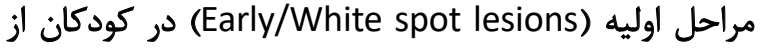
اولويت بالايى برخوردار بوده است [IT]

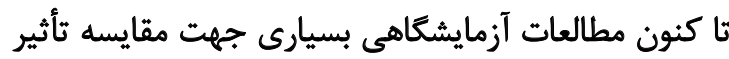

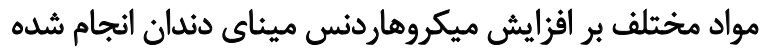

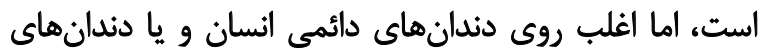
حيوانات صورت كرفتهاند.

از آنجا كه ميناى دندانهاى شيرى از لحاظ ساختار

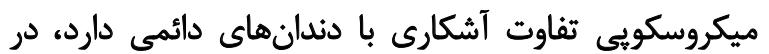

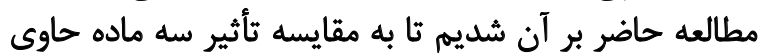

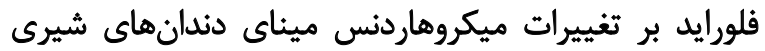

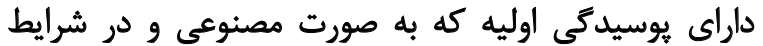
آزمايشكاهى ايجاد شدهاند، بيردازيمه

\section{مواد و روشها}

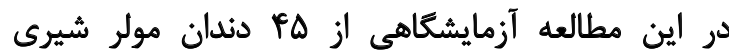

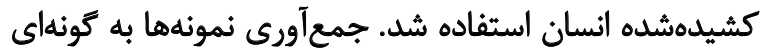

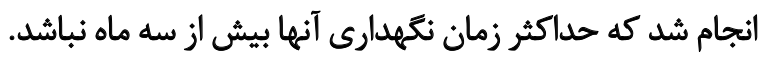

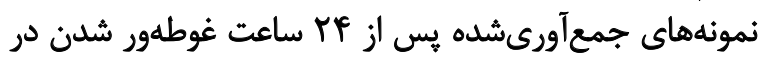

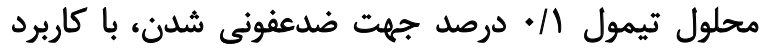

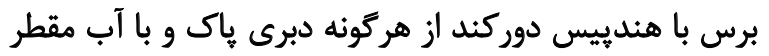

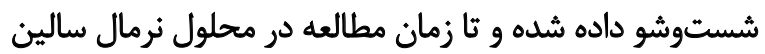

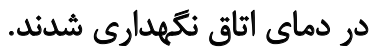

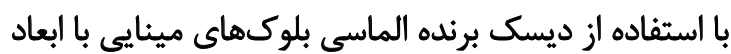

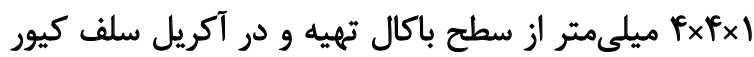

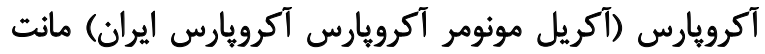

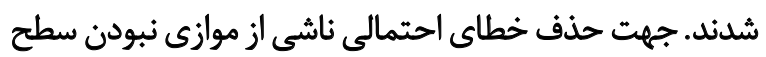

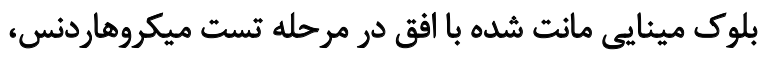

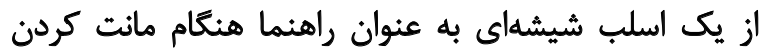

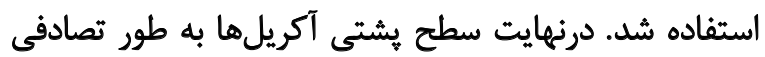

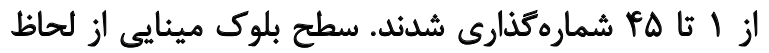

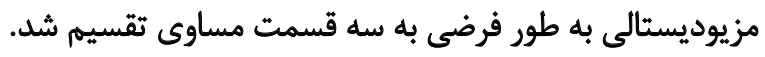
مرحله اول سنجش ميكروهاردنس: براى اندازهيرى إني

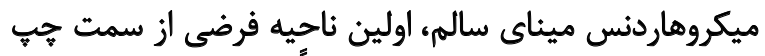

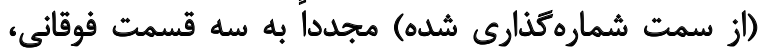
ميانى و تحتانى تقسيم شد (تصوير شماره (1). 


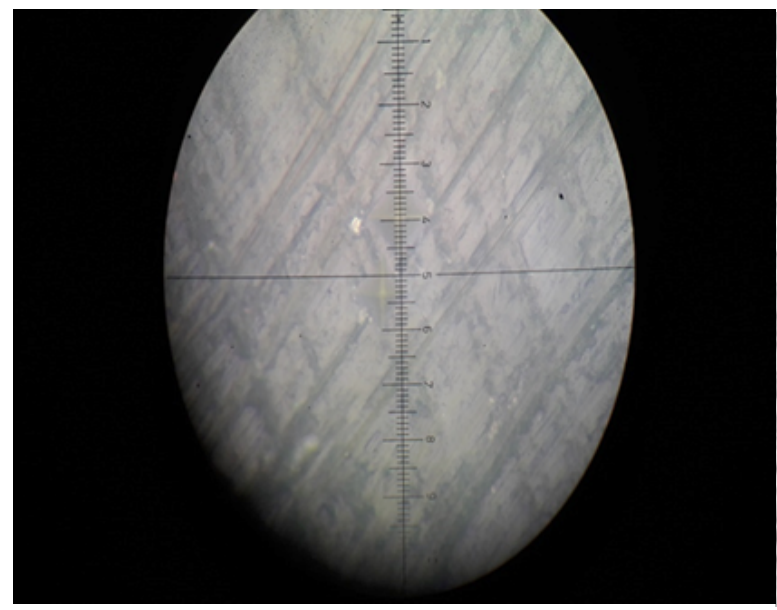
âns

مرحله سوم سنجش ميكروهاردنس: در اين مرحله در يك ديك

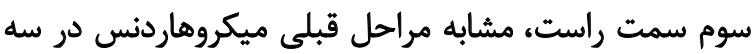

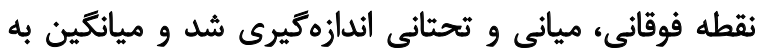

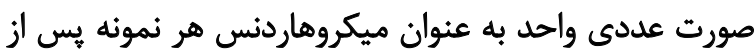

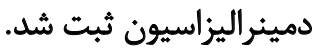

يافتهها

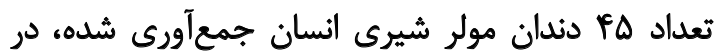

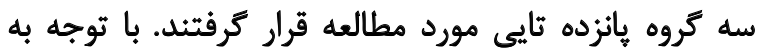

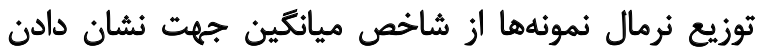
نتايج بهدست آمده در اين مطالعه استفاده شد.

ميانتينهاى ميكروهاردنس بين سه كروه وارنيش فلورايد،

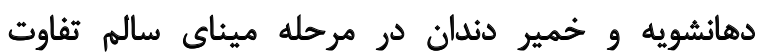

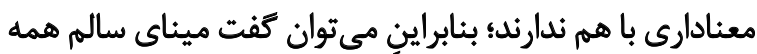

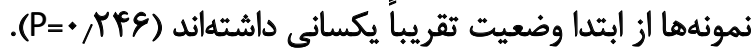
ميانكين ميكروهاردنس نمونههاى هر سه تروه مورد مطالعه

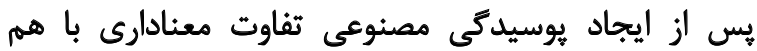

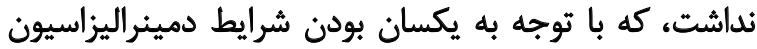

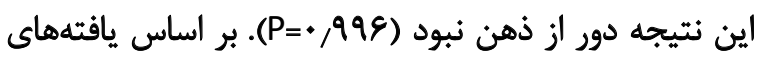

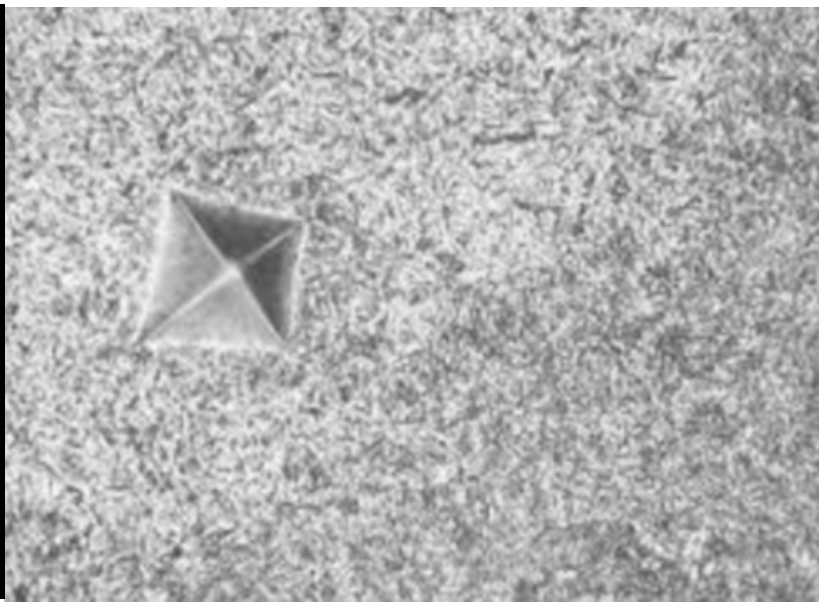

تصوير ا. تصاوير ميكروسكويى اندازميرى ميكروهارنس نمونهها درصد (V-Varnish, korea) با استفاده از ميكروبراش موجود

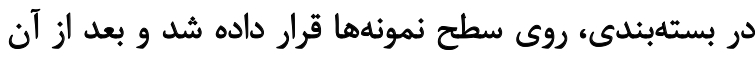

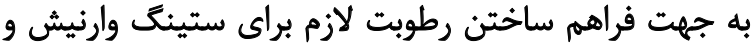

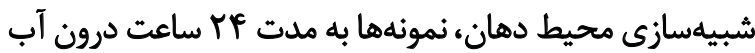

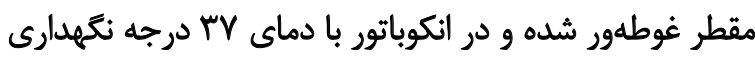

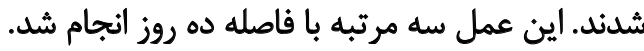

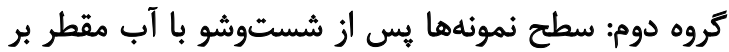
اساس دستور كارخانه سازنده با دهانشويه (Mouth wash) ما OralB مرتبه به مدت سى ثائيه غوطهور شده و اين عمل طر طى سله

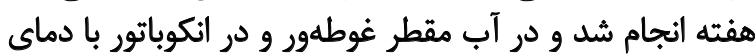

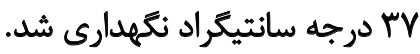

مروه سوم: روى سطح نمونهما يس از شيتوشو با آب

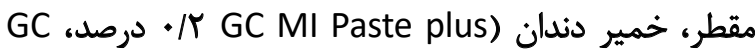

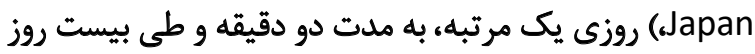

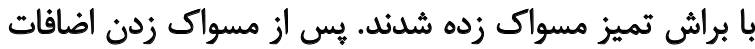

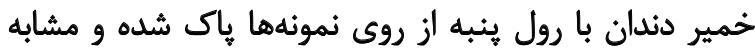

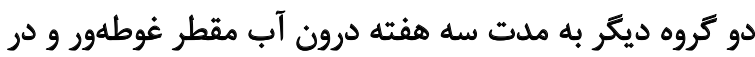

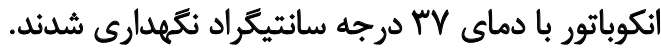

جدول ا. نتايج آناليز واريانس ميانكين ميكروهاردنس در سه مروه يس از اعمال فلورايد

\begin{tabular}{|c|c|c|}
\hline $\mathbf{P}$ & اختلاف ميانئين & أروها \\
\hline.$/ \cdot r$ & $-8 \cdot 194$ & وارنيش فلورايد دهانشويه حاوى فلورايد \\
\hline $.1 \% 19$ & $-881 \cdot r$ & وارنيش فلورايد خمير دندان حاوى فلورايد \\
\hline.$/ 1 \mathrm{rq}$ & $-\Delta / \kappa^{\epsilon}$ & دهانشويه حاوى فلورايد خمير دندان حاوى فلورايد \\
\hline
\end{tabular}




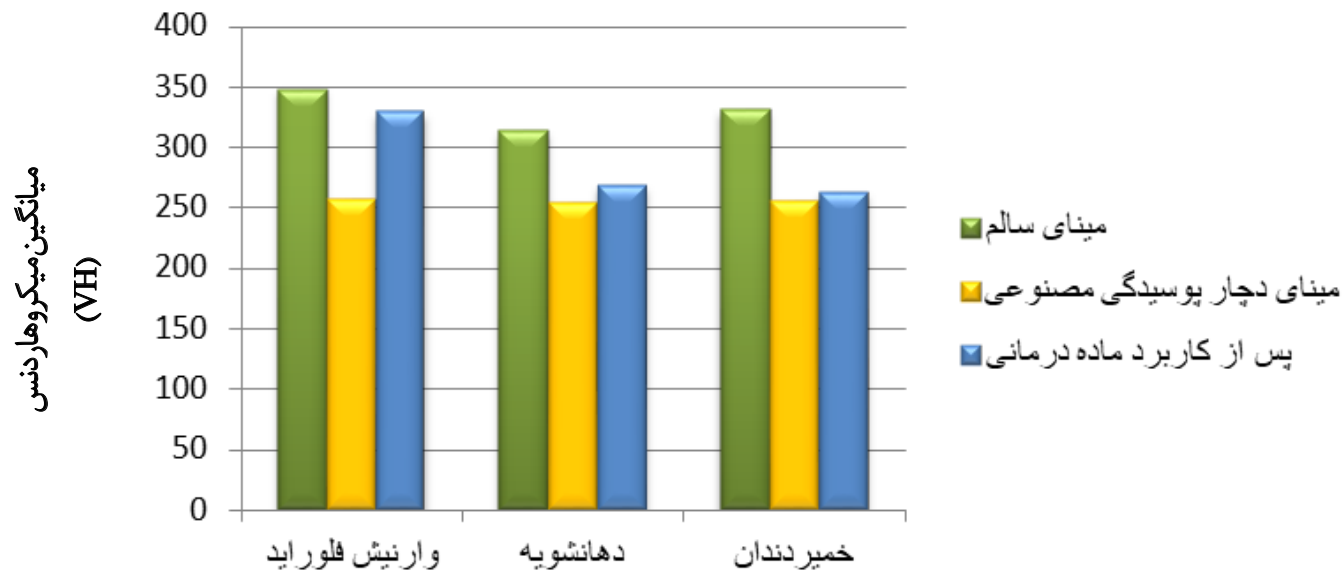

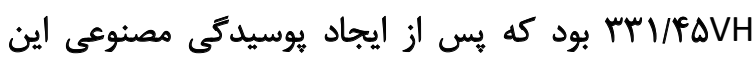

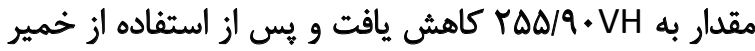

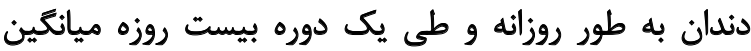

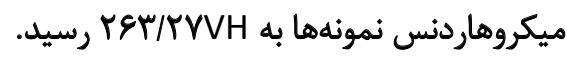

در اين گروه نيز مانند گروه دوم بين ميكروهاردنس

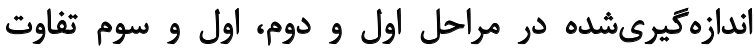

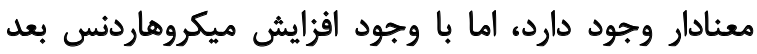

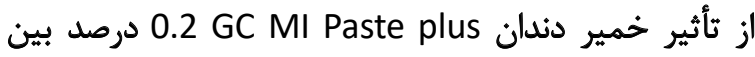
ميكروهاردنس اندازمخيرىشده در مران داحل دوم و سوم تفاوت

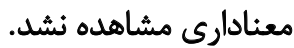

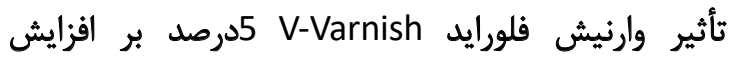

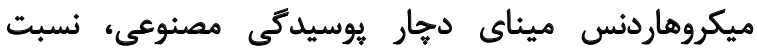

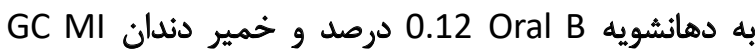
دPaste plus 0.2

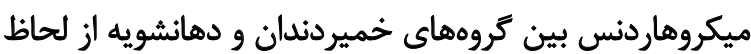

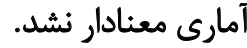

بحث

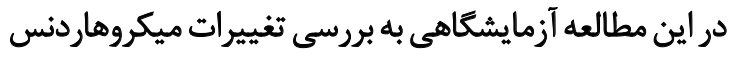

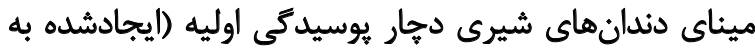

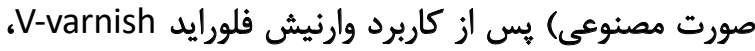

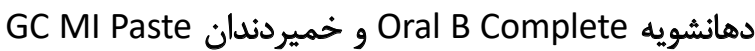

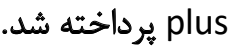

هدف از انجام سيكل دمينراليزاسيون و رمينراليزاسيون به

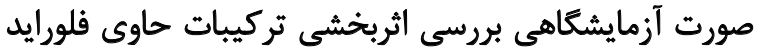

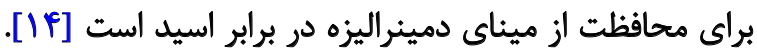

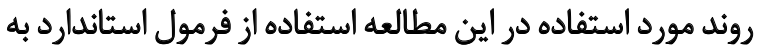

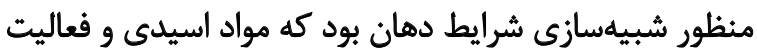

تصوير r. مقايسه ميانكين ميكروهاردنس كروههاى مختلف در سه مرحله سنجش

حاصل از مطالعه حاضر، ميانكين ميكروهاردنس (در هر سه

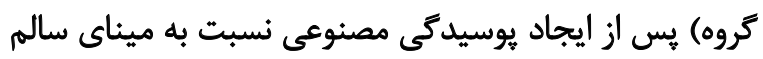

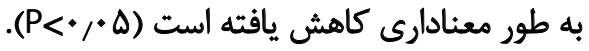

ميانكين ميكروهاردنس ميناي سالم در تروه اول مورد مطالعه

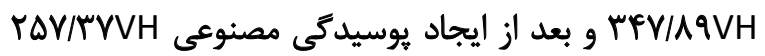

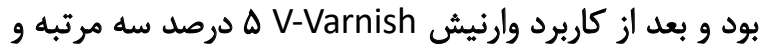

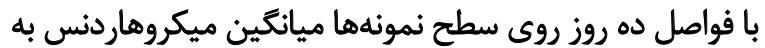

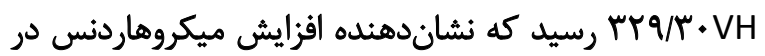

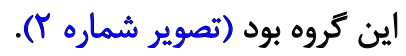

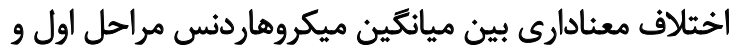

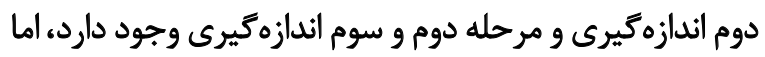

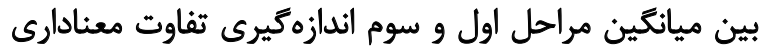

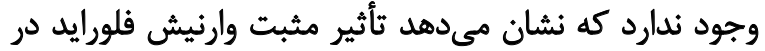

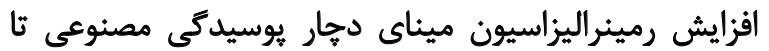

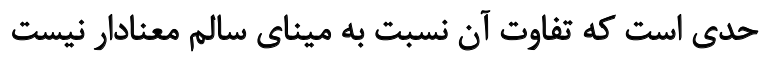
(تصوير شماره (r=./1r\&)

در كروه دوم مورد مطالعه، ميانكين ميكروهاردنس ميناى

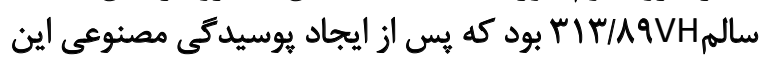

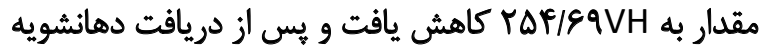

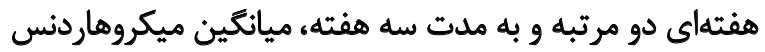

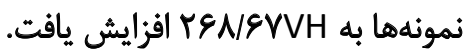

بين ميكروهاردنس اندازهيرىش مشده در مراحل اول و دوم و

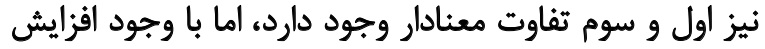

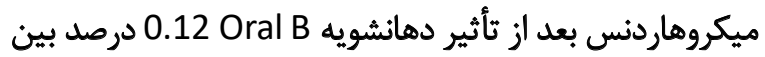

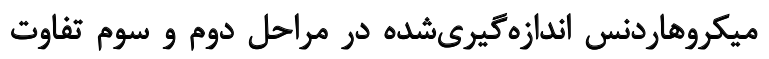
معنادارى مشاهده نشد.

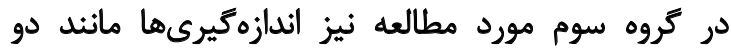

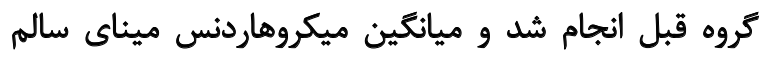


بودن غلظت فلورايد موجود در وارنيش نسبت به خمير دندان

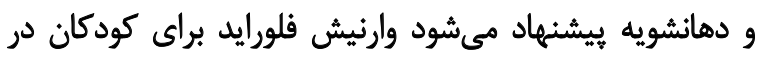

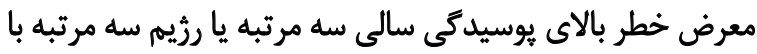
فواصل ده روزه طى يك مال يك استفاده شود.

همجنين با توجه به افزايش ميكروهاردنس ميناي دجار

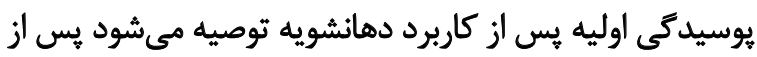
سن شش سالكى از دهانشويههاى حاري فارئ فلورايد استفاده شود. با وجود اينكه طبق مطالعه حاضر كمترين بهروديود

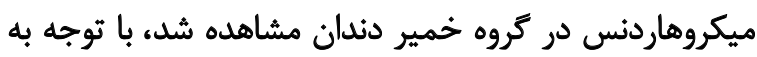

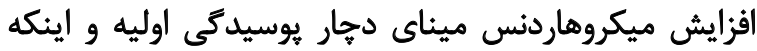

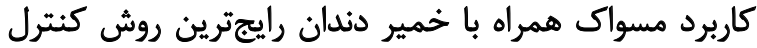

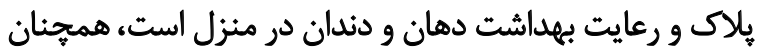

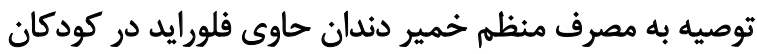

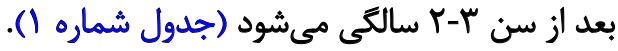

Losisting

ا. با توجه به كمتر بودن مطالعات در ارتباط با تأثير

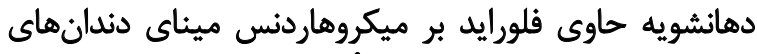

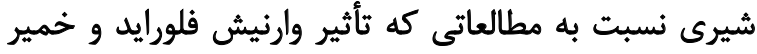

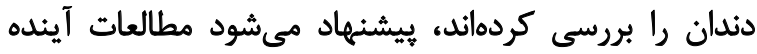

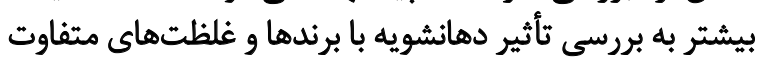

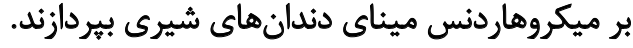

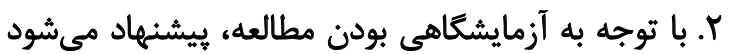

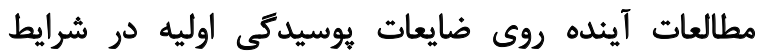

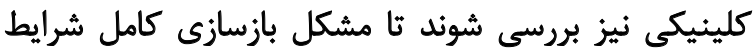

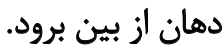

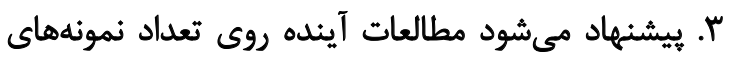

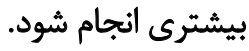

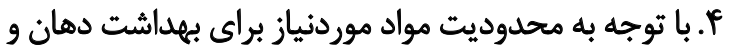

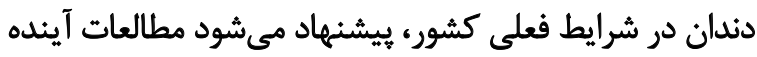
روى محصولات ساخت داخل كشور انجام شود.

اين مقاله از رساله دكترى نويسنده اول در تروه دندانيزشكى داني

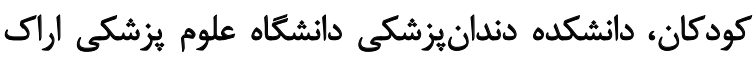

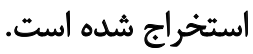
ماحظات اخلاقى

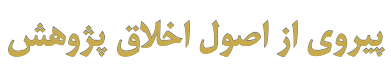
اين مطالعه با كد اخلاق 1 ائل

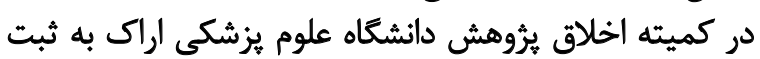
ريسه است.
باكترىهاى يوسيدگىزا باعث دمينراليزاسيون ميناي دندان و

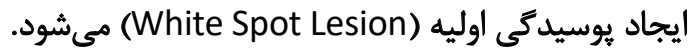
از آنجا كه مشخص شده است كه محتوا و نحوه انتشار فلورايد

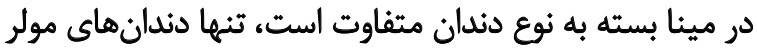

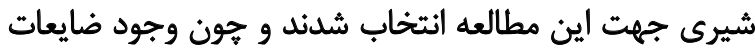

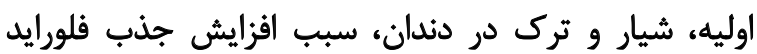

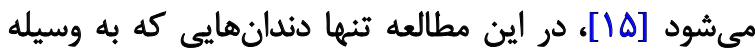

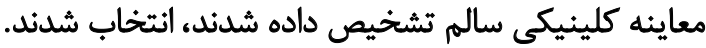
دليل ايجاد يوسيدگى مصنوعى در اين مطالعه اين بود

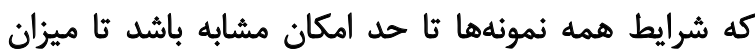

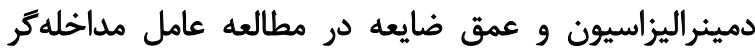

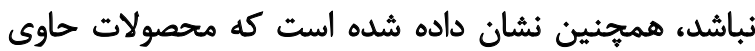

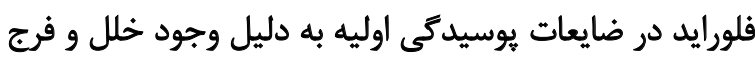

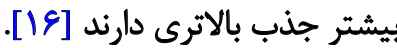

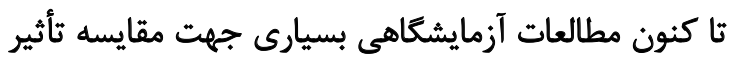

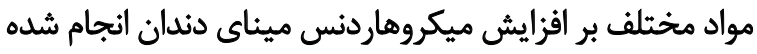

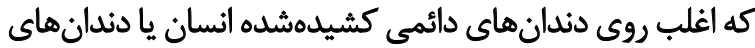

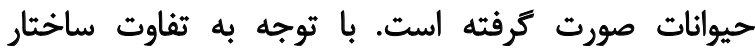

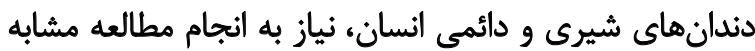

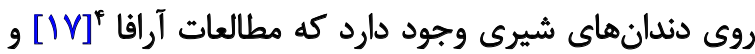

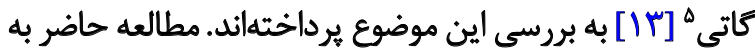
مقايسه مواد متفاوتى نسبت به مطالعات قبلى برائ برداخته است. در مطالعات آرافا و كاتي نيز از دندانهاي شيرى انسان كه

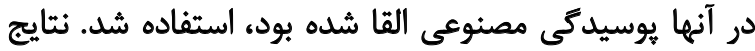

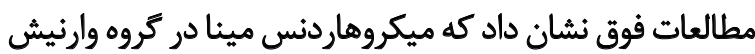

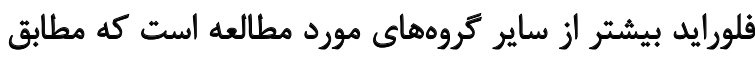

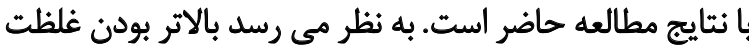

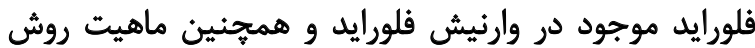

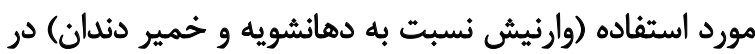

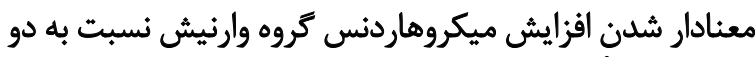
كروه ديكر تأثيركذار بوده است.

نتايج حاصل از اين مطالعه فرض مبنى بر عدم وجود تثفاوت

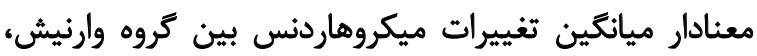
دهانشويه و خمير دندان را رد مى كنيد.

\section{تئيجليرى}

با توجه به ويزُكى هاي مطلوب وارنيش فلورايد، ازجمله كاربرد

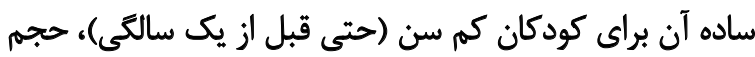

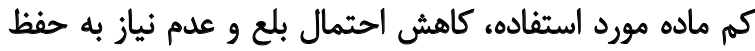

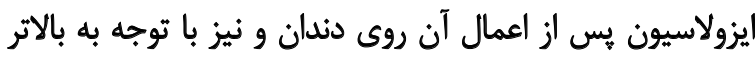




$$
\text { مالم }
$$

اين تحقيق هيج كونه كمك مالى از سازمانهاى ثأمين مالى نإي

در بخش هاى عمومى ، تجارى يا غير انتفاعى دريافت الفت نكرد.

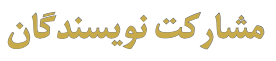

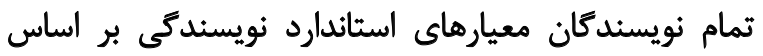

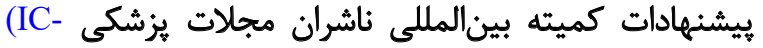

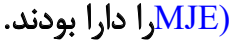

$$
\text { ثقارون منأقع }
$$

بنابر اظهار نويسندكان اين مقاله تعارض منافع ندارد. 


\section{References:}

[1] Taher NM, Alkhamis HA, Dowaidi SM. The influence of resin infiltration system on enamel microhardness and surface roughness: An in vitro study. Saudi Dent J. 2012; 24(2):79-84. [DOI:10.1016/j. sdentj.2011.10.003]

[2] Robinson C, Shore RC, Brookes SJ, Strafford S, Wood S, Kirkham J. The chemistry of enamel caries. Critical Reviews in Oral Biology \& Medicine. 2000; 11(4):481-95 [DOI:10.1177/10454411000110040601]

[3] Wang YL, Chang HH, Chiang YC, Lu YC, Lin CP. Effects of fluoride and epigallocatechin gallate on soft-drink-induced dental erosion of enamel and root dentin. J Formos Med Assoc. 2018; 117(4):276-82 [DOI:10.1016/j. jfma.2018.01.020][PMID]

[4] Nowak A, Christensen JR, Mabry TR, Townsend JA, Wells $\mathrm{MH}$ editors. Pediatric Dentistry-E-Book: Infancy through adolescence. Elsevier Health Sciences; 2018. https://books.google.com/ books?id=kXhaDwAAQBAJ\&dq=

[5] Zakizade M, Davoudi A, Akhavan A, Shirban F. Effect of resin infiltration technique on improving surface hardness of enamel lesions: $A$ systematic review and meta-analysis. J Evid Based Dent Pract. 2020; 20(2):101405. [DOI:10.1016/j.jebdp.2020.101405] [PMID]

[6] Hosoya Y, Marshall S, Watanabe L, Marshall G. Microhardness of carious deciduous dentin. Oper Dent. 2000; 25(2):81-9. [PMID]

[7] Dionysopoulos D, Tolidis K, Sfeikos T. Effect of CPP-ACPF and nanohydroxyapatite preventive treatments on the susceptibility of enamel to erosive challenge. Oral Health Prev Dent. 2019; 17(4):357-64. http:// www.quintpub.com/journals/ohpd/abstract.php?article_id=19692

[8] Creeth JE, Parkinson CR, Burnett GR, et al. Effects of a sodium fluoride- and phytate-containing dentifrice on remineralisation of enamel erosive lesions-an in situ randomised clinical study. Clin Oral Investig. 2018;22(7):2543-2552. [DOI:10.1007/s00784-018-2351-z]

[9] Kim MJ, Lee SH, Lee NY, Lee IH. Evaluation of the effect of PVA tape supplemented with \%2.26 fluoride on enamel demineralization using microhardness assessment and scanning electron microscopy: In vitro study. Arch Oral Biol. 2013; 58(2):160-6. [DOI:10.1016/j.archoralbio.2012.06.015][PMID]

[10] Rošin-Grget K, Peroš K, Šutej I, Bašić K. The cariostatic mechanisms of fluoride. Acta medica academica. Acta Med Acad. 2013; 42(2):179-88. [DOI:10.5644/ama2006-124.85] [PMID]

[11] Hiiri A, Ahovuo-Saloranta A, Nordblad A, Mäkelä M. Pit and fissure sealants versus fluoride varnishes for preventing dental decay in children and adolescents. Cochrane Database Syst Rev. 2010; (3):CD003067. [DOI:10.1002/14651858.CD003067.pub3][PMID]

[12] Mohammadi N, Farahmand Far MH. Effect of fluoridated varnish and silver diamine fluoride on enamel demineralization resistance in primary dentition. J Indian Soc Pedod Prev Dent. 2018; 36(3):257-61. [DOI:10.4103/JISPPD.JISPPD_4_18][PMID]

[13] Gatti A, Camargo LB, Imparato JC, Mendes FM, Raggio DP. Combination effect of fluoride dentifrices and varnish on deciduous enamel demineralization. Braz Oral Res. 2011; 25(5):433-8. [DOI:10.1590/ S1806-83242011000500010][PMID]

[14] Pancu G, Andrian S, lovan G, Ghiorghe A, Topoliceanu C, Moldovanu $A$, et al. Study regarding the assessment of enamel microhardness in incipient carious lesions treated by Icon method. Rom J Oral Rehab. 2011; 3(4):94-100. https://www.rjor.ro/study-regarding-the-assessment-ofenamel-microhardness-in-incipient-carious-lesions-treated-by-iconmethod/
[15] Huang GJ, Roloff-Chiang B, Mills BE, Shalchi S, Spiekerman C, Korpak $A M$, et al. Effectiveness of MI Paste Plus and PreviDent fluoride varnish for treatment of white spot lesions: A randomized controlled trial. Am J Orthod Dentofacial Orthop. 2013; 143(1):31-41. [DOI:10.1016/j.ajodo.2012.09.007][PMID][PMCID]

[16] Cury JA, Andaló Tenuta LM. Enamel remineralization: controlling the caries disease or treating early caries lesions? Braz Oral Res. 2009; 23(Suppl 1):23-30. [DOI:10.1590/S1806-83242009000500005][PMID]

[17] Arafa A. Synergetic remineralization effectiveness of calcium, phosphate and fluoride based systems in primary teeth. Pediat Dent J. 2017 27(1):65-71. [DOI:10.1016/j.pdj.2016.12.003] 
This Page Intentionally Left Blank 